\title{
Estructura y fundamentos de los laboratorios de investigación empresarial para la estrategia y la gestión organizacional en Colombia ${ }^{1}$ Structure and fundamentals of research laboratories business strategy and organizational management in Colombia
}

Recibido: 9 de abril de 2013

Evaluado: 19 abril de 2013

Aceptado: 1 julio de 2013

\begin{abstract}
Jahir Alexander Gutiérrez Ossa (Colombia)
Universidad CES

Economista, Universidad de Medellín, Colombia; Ph.D en Administración Pública, Atlantic International University (AIU), Honolulú, Estados Unidos; magíster en Desarrollo, Universidad Pontificia Bolivariana, Medellín, Colombia; estudios en Sistemas de Información Geográfica, Universidad San Buenaventura (USB), Medellín, Colombia; investigador, Facultad de Ciencias Administrativas, Universidad CES - Universidad del Rosario, Medellín, Colombia; investigador y líder del Grupo de Gestión Empresarial del CES y miembro del Grupo de Perdurabilidad Empresarial, Universidad del Rosario.

algutierrez@ces.edu.co
\end{abstract}

\section{Rubén Darío Manrique Hernández (Colombia) \\ Universidad CES \\ Químico farmaceuta de la Universidad de Antioquia, Medellín, Colombia; Ph.D en Epidemiología, Universidad de Antioquia; magíster en Epidemiología, Universidad de Antioquia; especialización en Altas Técnicas del Laboratorio Forense; investiga- dor, Grupo de Evaluación de Tecnologías en Salud, Universidad CES; director de la Unidad de Gestión de Conocimiento, Universidad CES, Medellín. \\ rmanrique@ces.edu.co}

1. Este documento es fundamento para la formulación del Laboratorio de Investigación Empresarial en la Universidad CES, producto del proyecto de investigación "Competencias investigativas para empresas (empresarios y profesionales) desde la relación Universidad, Empresa y Estado (UEE) en el caso Colombiano", financiado por la Dirección de Investigaciones de la Universidad CES (Medellín) - Universidad del Rosario (Bogotá), en el período octubre de 2010 a febrero de 2011, llevado a cabo por el Grupo de Investigación en Gestión Empresarial de la Facultad de Administración CES, registrado en Colciencias, y el Grupo de investigación en Perdurabilidad Empresarial (GIPE), categoría A1, de la Universidad del Rosario. 


\section{Resumen}

Este escrito plantea la conveniencia de estructurar y fundamentar los laboratorios de investigación empresarial, a partir de los hallazgos y las pesquisas desarrolladas por los grupos de investigación en Colombia. Al margen de la vinculación, la expectativa creada entre ambos actores no termina con la consecución de publicaciones y la finalización de la investigación. Los laboratorios de investigación empresarial en sus diferentes concepciones y versiones afianzan la relación universidad y empresa, ubicando a esta última en el centro del proceso; etapa que pasa luego al laboratorio para analizar hechos e intereses desde distintos ángulos y así continuar desarrollando campos y temas concretos de interés empresarial. Para dar sentido a la investigación es necesario disponer a las empresas efectivamente los hallazgos y resultados frutos del acercamiento.

Palabras clave: comportamiento de la empresa, elección intemporal de la empresa y crecimiento, estrategia y gestión organizacional, laboratorios de investigación empresarial.

\section{Abstract}

This paper raises the need to structure and consolidate corporate research laboratories, from the investigation findings and developments done by research groups in Colombia. Regardless of their connection, the expectation created between both actors does not end with the attainment of publications and the completion of the investigation. Corporate research laboratories in different versions and conceptions strengthen the University - enterprise relationship, placing the latter in the center of the process. This stage moves on to the laboratory to analyze facts and interests from different angles, and so continue to develop fields and specific topics of business interest. To give sense to research it is necessary to effectively share the findings and results derived from this approach.

Keywords: behavior of the company, timeless choice for enterprise and growth, strategy and organizational management, corporate research labs. 


\section{Introducción}

La investigación de carácter empresarial permite orientar el quehacer de los investigadores dedicados a dichas pesquisas. Sin embargo, más allá de las razones puntuales que animan el interés, no es evidente el reconocimiento de un laboratorio de investigación empresarial, por lo menos en Colombia, que además de destacar los hallazgos obtenidos por diferentes instituciones destaque una labor constante en materia de transferencia de impactos, resultados o servicios a las empresas desde las que ha de extenderse un interés concreto y permanente.

El escenario que ha atendido el margen de maniobra de las universidades y empresas ha corrido por cuenta de la presencia de estamentos dedicados a la consagración de la relación universidad, empresa y Estado (UEE). No obstante, cuando se evalúa el papel que debe cumplir cada unidad de la tríada se reconoce el quehacer externo, pero es muy poco lo que se trabaja en el interior para que efectivamente los esfuerzos, productos y resultados obtenidos en cada investigación superen la etapa marcada en cuanto a obtención de productos.

La puesta en escena de los laboratorios de investigación empresarial expone la necesidad de identificar el recorrido que debe tener todo proyecto, cuando efectivamente impacta y es útil. Mientras los proyectos de investigación no alcancen a ser internalizados, cualquier acercamiento vinculado a ello tendrá solo el fundamento de un trabajo alegórico, descriptivo o especulativo. La investigación desarrollada por los grupos académicos de las universidades debe alcanzar una rigurosidad superior a la obtenida con los productos conseguidos por medio de los laboratorios.

Es relevante llevar la investigación de interés empresarial, de la sola compilación de información y estructuración de datos, a un nivel superior. El escenario radica en poder convalidar, en consecuencia, que el proyecto inicialmente debe pasar por etapas de revisión experimental y de observación con el propósito de establecer la idoneidad de la investigación o la posibilidad de que esta sea llevada hacia otra escala, a favor de fortalecer, en consecuencia, los resultados para las empresas, paso que no han seguido los proyectos concluidos.

En la mayoría de los estudios e investigaciones las empresas terminan siendo solo objeto de análisis en una parte del proceso, cuando son las que representan el origen, cometido y resultado del trabajo a saber. El aparente estado de aislamiento al que son sometidas por diversas razones, no siempre explicadas o sustentadas, retrasa la posibilidad de que efectivamente, cuando se analiza y evalúa alguna actividad empresarial o la organización en pleno, por momentos o en la estrechez de un cuestionario, pueda obtenerse una investigación completa, plena y útil.

Con la finalidad de explicar las bondades y sustentos de esta propuesta, la estructura del artículo obedece a la siguiente composición: en primer lugar, el análisis que comporta la estructuración del laboratorio empresarial, en términos generales; en segundo lugar, los ejemplos que han rodeado a las diversas instituciones y grupos de investigación que han asumido con trascendencia la iniciativa; $y$ finalmente, las consecuencias lógicas que puede traer la intervención empresarial 
desde allí. Es necesario que los grupos trasciendan de la investigación formativa a la aplicada.

\section{Estructura y formulación general del contexto de laboratorio para el análisis empresarial}

El patrón característico de las investigaciones, particularmente en el ámbito académico-empresarial, no supera el talante de diagnóstico, precepto que desdibuja la generación de impacto en las empresas. El análisis obtenido mediante los diversos mecanismos utilizados para obtener datos y compilar información carece en la mayoría de los casos de disertación, contravención o rastreo, dirigido precisamente a elevar los componentes explicativos de la investigación desde los fundamentos provistos en los laboratorios de investigación empresarial. Conceptúa Miyahira (2009, p. 119):

¿Qué se entiende por investigación? La Real Academia de la Lengua Española define investigar a realizar actividades intelectuales y experimentales de modo sistemático con el propósito de aumentar los conocimientos sobre una determinada materia. Entonces, se considera investigación desde la indagación sistemática y organizada hasta la práctica experimental a través de la cual se genere nueva información y posterior conocimiento. El carácter de la investigación empresarial exige elevar el nivel de trabajo académico e investigativo que implica para las partes, universidad y empresa, el disponer recursos para que los resultados en ambos frentes obedezcan a una sana lógica. Las empresas esperan obtener elementos de trabajo con los cuales poder avanzar, mejorar o tomar decisiones que representen réditos concretos, y los grupos de investigación, el poder identificar los elementos de trabajo que les son propios, y que efectivamente traen beneficios importantes para su continuidad. Establece Casanovas: "En el ámbito empresarial la opinión general relevada es que en su formación se enfatiza el desarrollo de habilidades técnicas y poco el análisis de problemas, escenarios y selección de herramientas de solución adecuadas (toma de decisiones)" (2005, p. 18).

No ha sido establecido el puente que permita establecer lazos recíprocos entre la gestión hecha por parte de las universidades y los grupos de investigación, para interiorizar la etapa de mercado al que están expuestos a la hora de ejecutar o presentar un proyecto de investigación, que en sus parámetros contempla la necesidad de acercarse o tomar referencia discrecional sobre ella. La dilación de los proyectos radica precisamente en que no se sabe claramente el destino de los resultados, más allá de la necesidad de publicarse o refrendarlos en eventos. Exponen Moreno et al. (2009, pp. 33-34):

Si en muchos países los vínculos entre la investigación universitaria y las actividades industriales son débiles, en nuestra región el problema es mucho peor. Para ampliarlas y orientarlas más directamente al crecimiento, se requiere de un sistema nacional de innovación que comprenda tres factores esenciales: a) recursos humanos (personal técnico y de investigación); b) infraestructura 
adecuada (laboratorios, talleres, equipo de cómputo, bibliotecas); y c) instituciones que vinculen a los grupos de académicos de investigación de las universidades con las empresas que producen bienes y servicios para el mercado. Los vínculos universidad-industria en los países desarrollados no fueron creados al azar, o por las fuerzas del mercado solamente; en muchos de ellos hubo una acción deliberada del Estado, como asunto de política de Estado (Yusuf, 2007) con el fin de promover una relación mutuamente benéfica entre los centros de investigación (usualmente las universidades) y las empresas privadas (y públicas) en muchas ramas de la economía.

Las instituciones de educación superior y universidades deben reconocer el papel de formadoras de carácter académico que hasta ahora las ha caracterizado. Sin embargo, el esfuerzo dirigido a investigación debe contener los criterios básicos sobre los que efectivamente esta debe ser desplegada. Los resultados de investigación deben pasar lógicamente a la gestión administrativa de las instituciones, pero también, deben constituirse en puntos de referencia para que las instancias pertinentes conviertan los resultados en oportunidades para todos.

Considera el Instituto Internacional de la Unesco para la Educación Superior en América Latina y el Caribe (2008, p. 135):

Somos universidades, instituciones de educación superior latinoamericanas y caribeńas. Queramos o no. No somos Harvard. Somos latinoamericanas y caribeñas. Pero queremos caminar hacia la excelencia de Harvard. Queremos una universidad excelente, pertinente, ubicada y contextualizada. Yo creo que esta es una propuesta política que tenemos que enfrentar. El mapa va a decir con toda claridad que nuestras universidades tienen una acción compleja, que nosotros consideramos pertinente e importante. Entre sus dimensiones está la excelencia, que es la meta de todos nosotros. Nuestro reto es mantener una doble lealtad con la universalidad del conocimiento y con la pertinencia, el ser y estar aquí y ahora mirando hacia el futuro.

Es la discrepancia, la poca formalización y la escasa posibilidad de hacer seguimiento al proceso de investigación en las organizaciones, el que ha llevado a considerar el hecho de que las empresas en esencia no hacen parte del cometido e interés por quienes se acercan desde distintos frentes para sacar de ella información o datos, para llevar a resultados de investigación o productos fruto de esta. Tal es así, que no es claro definir el alcance que puedan tener las investigaciones en las empresas, luego de concluir. Explica Pérez (2009, p. 178):

En virtud de que las universidades no pueden asumir todos los costos de la innovación y deben acudir a la ayuda de los fondos empresariales, ha habido cambios en la rutina gerencial de las dos instituciones. Las primeras aportan conocimiento con el concurso de sus investigadores, y las empresas celebran contratos en proyectos de innovación, con lo que agregan valor a la producción.

La no existencia de protocolos de avance y evaluación de resultado de las 
empresas y de los grupos de investigación, al no avanzar de manera concreta para con las expectativas trazadas, constituye el patrón característico de dicha relación. Las empresas plantean expectativas que no son filtradas en los proyectos de investigación en ninguna de sus etapas. La empresa pasa por una especie de aislamiento provocado desde la propia etapa de formulación del proyecto y contradictoriamente, durante el desarrollo y finalización del proyecto. Reitera Renzulli (2010, p. 36):

Este tipo de aprendizaje contrasta con la cadena de sucesos que tienen lugar en los laboratorios de investigación, en las oficinas de negocios o en los estudios cinematográficos. En estas situaciones, el objetivo es originar un producto o servicio. Todos los recursos, la información, los horarios, los acontecimientos, se dirigen hacia el logro de este objetivo. Así pues, buscar nueva información, conducir experimentos, analizar resultados o preparar un informe son actividades enfocadas principalmente hacia la necesidad inminente de obtener información inmediata más que para un futuro distante. Es este uso que hacemos de los aprendizajes inmediatos (presentes) lo que confiere un mayor valor a la transferencia para un uso futuro.

El interés de llevar las investigaciones al registro de laboratorio representa una de las respuestas que deben ser ofrecidas a la hora de refrendar el sentido de la investigación. Al respecto, no solo los grupos y universidades deben estar preparados para afianzar dicho trabajo, las empresas han de convencerse respecto a la fuerza e impacto que puedan tener estos acercamientos, siempre y cuando admitan la necesidad de establecer lazos de cooperación y reciprocidad, que implica explícitamente revisar por parte de ellas el alcance del trabajo a saber.

Plantea Segarra (2010, pp. 55-56):

Las ideas pueden tener diversos orígenes (existen diferentes mecánicas que las empresas pueden seguir para hacerse con ellas), pero lo que me gustaría destacar es que, independientemente de cuál sea el modo predominante para obtenerlas por el que opte la empresa, lo importante es que seamos capaces de tender puentes entre esas cosas que nos llegan del laboratorio o de nuestros empleados/colaboradores y nuestro objetivo comercial.

Es la oportunidad de reiterar el compromiso e interés para que las conclusiones e impactos obtenidos en cada etapa del estudio e investigación tengan un aliciente superior, que la sola complacencia por alcanzar los objetivos aprobados y preestablecidos. De allí, que el tema relacionado con la gestión del conocimiento y la negociación a la que haya lugar, tenga un sentido más claro. En las empresas estriba la posibilidad de que los proyectos de investigación llevados al escenario de laboratorio tengan un importante grado de éxito e impacto. Expone Ondagueti (2001, p. 117):

Hacer ciencia e innovar en garajes no será propio del siglo XXI. Esto presupone una masa crítica de empresas, agentes e individuos trabajando en sectores y actividades determinadas y un clima de optimismo temporal o cíclico, en el cual las ideas y sus aplicaciones afluyen y se suceden con rapidez, produciéndose una reacción en cadena que no 
solo es necesario valorizar, sino que además es necesario volver a innovar.

El campo de acción que puedan tener las partes involucradas no implica necesariamente una alianza a largo plazo, pero sí la definición de un trabajo que bajo la naturaleza que se pretende promover, exige coherencia entre lo que se estima como investigación con fines académicos e investigativos, con la que va dirigida precisamente a considerar problemáticas puntuales de las empresas o del contexto que impactan en las perspectivas de estas y que claramente plantean para ellas la posibilidad de contar con equipos de trabajo relacionados. De nuevo analiza Segarra (2010, p. 55):

La última fuente de ideas y quizá la que está dando más que hablar es la llamada "innovación abierta", que consiste en abrir la puerta a todo aquel que quiera participar —aportando ideasen proyectos de innovación auspiciados por las empresas. Aun cuando está todavía en estado embrionario, puesto que no existe suficiente experiencia para valorar resultados reales, ya existen fervorosos entusiastas y escépticos a partes iguales.

Es el desempeño estructural y escalamiento de los procesos de investigación ejecutados por los grupos de investigación el que puede orientar la disposición de laboratorios de investigación empresarial, lo que también se convierte en una constante en un mundo para el cual es importante que de manera permanente no solo se evalúen problemas, sino que con ellos puedan plantearse alternativas para quienes están inmersos en dificultades, labor que solo puede refrendarse en la medida en que se atienda a la empresa como un sujeto de análisis permanente. Plantea Galán (2004, p. 142):

En este sentido, existe una gran cantidad de estudios empíricos centrados en el análisis de varios aspectos relevantes sobre los procesos de cambio estratégico y organizativo experimentados por las organizaciones a lo largo del tiempo. Sin embargo, como correctamente han apuntado muchos investigadores, la mayor parte de estos estudios, salvo notables excepciones, presentan básicamente un carácter estático, transversal, aprocesual, acontextual y ahistórico. Desde este punto de vista, recientemente emerge una opinión creciente de que la investigación en este campo de estudio debe cumplir, en la medida de lo posible, con el doble requisito de rigor académico y de relevancia práctica para la dirección de las empresas. De este modo, existe un interés cada vez mayor en que los estudios sean más reales, con el fin de que proporcionen consejos y/o ideas útiles para los directivos y la práctica empresarial en general. Ello requiere, además de indagar sobre el estado del arte, compasarse de la mano de las propias empresas, para que revelen información que pueda arrojar luz sobre las cuestiones a investigar. Así los procesos en esta área de estudio tienden a insertarse en la investigación de proceso (process research).

La gestión del conocimiento establece un puente importante a la hora de conjugar el vaciamiento dado en los proyectos de investigación con el proceso que debe darse a medida que dichas actividades afianzan los cometidos. En este sentido, se esgrime la prioridad de provocar permanentemente para que los grupos sean guiados bajo la idea de generar, además 
de nuevo conocimiento, impacto para con el objeto o sujeto de estudio, a los fines de adquirir el carácter de centro estratégico, laboratorio o tanque de pensamiento. Explica el Instituto Internacional de la Unesco para la Educación Superior en América Latina (2008, p. 69):

La universidad desde sus inicios es el lugar donde se crea conocimiento y se lo transfiere a la sociedad. Esa es su misión esencial: crear y transferir. El aspecto docente es algo que necesariamente viene adosado a tal proceso. Un lugar donde solo se cree conocimiento y no se lo transfiera puede ser algo muy interesante y maravilloso, pero no es una universidad en el sentido de Stanford, Berkeley, Oxford, o Cambridge.

Es importante, para lograr certeza en el proceso, que las empresas reconozcan en dichos ejercicios la bondad de continuar trabajando con diversas instituciones, y de ello se espera que terminen afianzando, además de sus intereses, la proyección que tienen como organización. En consistencia con dichos propósitos, el escenario del laboratorio de investigación radica precisamente en elevar las condiciones sobre las que se argumenta, analiza y concluye respecto a las revisiones dadas en el ambiente académico con relación a la empresa. Esgrime Renzulli (2010, pp. 35-36):

Cualquier cosa que resulta del aprendizaje en un laboratorio de investigación es de uso inmediato. Así pues, buscar información nueva, hacer experimentos, analizar los resultados o preparar un informe son tareas enfocadas al presente más que al futuro. Incluso la cantidad de tiempo dedicada a un proyecto particular no puede estar determinada o fijada de antemano, porque la naturaleza del problema y los obstáculos desconocidos que podemos encontrar previenen contra horarios-calendarios predeterminados de forma rígida.

El laboratorio empresarial permite la continuidad de los resultados iniciales obtenidos en cada proyecto. Su particularidad radica en enriquecer el ejercicio, los logros y productos generados durante el proceso, precisamente, articulándolos a la gestión de investigación que corre por cuenta del área o dirección de investigación, para la cual, al instalar los trabajos como fuente de generación de asesorías, consultorías, estudios, deben igualmente contextualizarse como etapas que debe cumplir eficazmente todo proyecto de investigación que sea serio. Expone Fracica (2009, p. 62): "Desde la perspectiva de los empresarios se demanda una masa crítica de proyectos de base tecnológica y de rápido crecimiento”.

\section{Estructura aplicada y formal de los laboratorios de investigación empresarial}

La constitución del laboratorio refrenda la concepción genérica que se tiene de él, como un espacio donde se desarrollan actividades con el propósito de obtener evidencias o hacer pruebas conforme al objeto de estudio analizado, y las condiciones en que este se presenta en diferentes etapas. En cada una de ellas se presentan diversos momentos que conducen a la 
revisión permanente de datos e información respecto a los pasos dados en cada etapa. Es decir, que en el laboratorio se intenta afianzar el contenido inicial del estado actual de la investigación. Amplía Canada (2010, p. 42):

Su enfoque de reunir expertos para solucionar su problema ha sido alabado mundialmente como un beneficio de utilizar un "enfoque de innovación abierta”. En resumen, crearon un mapa centrado en hallar valor, en lugar de centrarse en verificar los datos existentes y, a lo largo del proceso.

La necesidad de tomar referencias con cada prueba ejecutada y realizada a la par con los criterios obtenidos con los últimos avances, constituyen, a los efectos, el ejercicio inicial de revisión lógica del contenido y de los resultados alcanzados en dicha etapa, que deben ser igualmente probados. Es en dicho espacio en el que las hipótesis y las metodologías ilustradas en la etapa de formulación del proyecto de investigación empresarial toman fuerza y medida, con relación a las distintas interacciones que se pueden acusar o incentivar en el ámbito del laboratorio. Agrega Marzo et. al. (2008, p. 50):

Por lo tanto, los gestores de las instituciones universitarias, a diversos niveles y en función de sus competencias, ministerio, rectores, decanos, fundaciones universidad-empresa, deberían analizar los factores que determinan la satisfacción de las empresas en sus relaciones con las universidades, ya que esta es un elemento clave para obtener una participación voluntaria de las empresas, tal y como se ha verificado empíricamente en este trabajo.
El proyecto de investigación empresarial que converge a las empresas y a los grupos de investigación está dividido en dos grandes apuestas. La primera, vinculada con la perspectiva que imprime en él quienes deciden emprender el proyecto conforme a criterios y fuentes de diversas corrientes, sustrato que explícitamente termina siendo un punto de reconocimiento para los grupos con referencia al entorno y de las empresas que aspiran a analizar. La segunda etapa, corresponde a ese nivel de criterios que insta las bases de análisis del laboratorio. Agrega Svarzman (2007, p. 43):

Adicionalmente a ello, diseñar e implementar herramientas orientadas a "acercar" al mundo de los negocios con el de la investigación y el conocimiento científico (universidades, centros de desarrollo públicos y privados, etc.), generando al mismo tiempo incentivos específicos para que ambas partes perciban como necesario y provechoso el estrechamiento de su capacidad de interacción y de sus vínculos económicos, humanos e institucionales, constituye uno de los principales desafíos que enfrentan nuestras sociedades de cara al próximo decenio, a efectos de encontrar un modelo de desarrollo económico y socialmente sustentable.

En este escenario, se llega al cumplimiento de los objetivos trazados en la intención del proyecto, pero no en su terminación con la inclusión de un mapa de oportunidades. La fundamentación e ilustración provista por diferentes fuentes y estamentos obedece a la construcción del estado de la cuestión que no alcanza a ser sometido a prueba, debido 
precisamente a que el papel que cumple es el de orientador en principio de ideas, para luego de pasar por análisis previos terminar por permitir de cierta forma que sean ordenadas algunas ideas, pero que tampoco tienen término para ser revisadas bajo el carácter de prueba. Refrenda Canada (2010, p. 43):

Mapa de oportunidades: una representación visual del entorno de la empresa que guíe la estrategia futura al sintetizar las necesidades de los clientes satisfechas y las no satisfechas; las discontinuidades emergentes, como tendencias culturales y tecnológicas; la actividad competitiva tanto directa como indirecta; y las competencias que son competitivas, tanto internas como externas. Sintetizar todos estos datos ayuda a los equipos de innovación a separar aquellas oportunidades en las que deberían centrarse de las docenas en las que no deberían hacerlo. Aunque no hay reglas absolutas respecto a cuántas capas de datos se deben incluir o qué tipo de datos son más importantes en un mapa de oportunidades.

El poder determinar que solo han sido alcanzados los objetivos prestablecidos al comienzo de la investigación, es una etapa que obedece al contexto e ilustración del marco general o referencial, que termina de cierta manera en un proceso de actualización para quien lo ubique en un estadio de tiempo particular. De allí, deben aparecer otros objetivos, si es que se cuenta con los recursos y planteamientos para lograrlo y, son estos los que efectivamente terminan siendo de interés para las partes en una etapa de negociación. Sin embargo, este vaciado responde en la medida en que se atienda la flexibilidad del proyecto (ver cuadro 1 ).

Cuadro 1. Ventajas de la relación

entre universidad y empresa

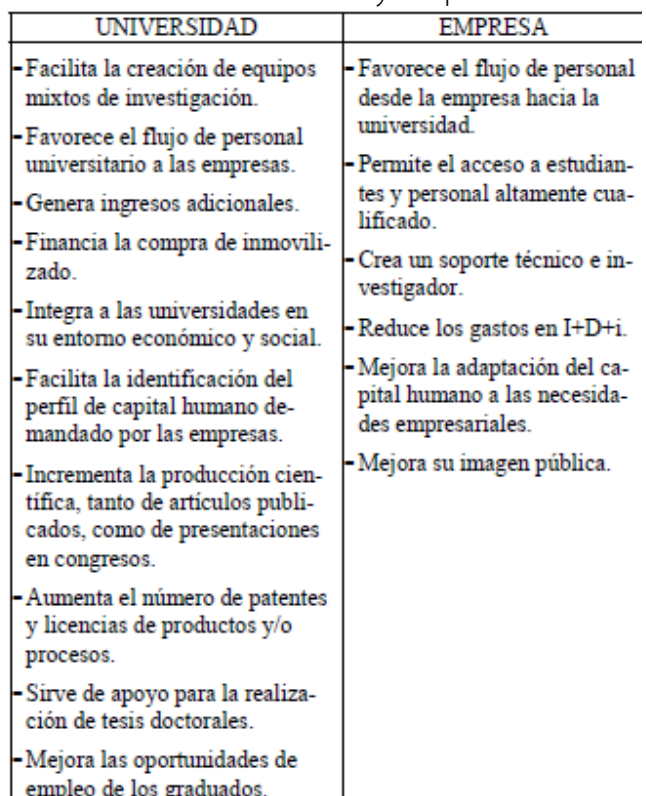

Fuente: Elaboración propia a partir de Barnes et al. (2002), Fernández et al. (2004), Fombrun (1996), García et al. (2003), González (2000), López et al. (2000), Santoro (2000) y Solé (2004).

En: Marzo et al. (2008, p. 42).

En cuanto a las hipótesis y metodologías que terminan siendo la puerta de entrada para ubicar el proyecto de investigación empresarial en el proceso de laboratorio, estas pueden desarrollarse paralelamente a la etapa que inicia con la construcción de los últimos avances. La refrendación de este aparte no puede convertirse en el punto de referencia para interactuar con las empresas. Al contrario, constituye el ámbito de aditamentos que debe tener presente para ir construyendo 
el camino de análisis que posibilite la revisión empresarial de ello. Expresan Rodríguez y Betancur (2009, p. 58):

El acercamiento de los empresarios con la academia ya es un referente de la manera como los gremios han asumido su compromiso con los procesos de formación de los futuros profesionales. Este no es el único aspecto que cabe mencionar, ya que el ejercicio mismo de transversalidad, que realizan los equipos de estudiantes con el acompañamiento de los docentes, aporta un diagnóstico empresarial, que ha permitido tener registrados los resultados de los trabajos finales que ellos han presentado en varias empresas.

Es importante que se haya entendido que todo proyecto de investigación debe ser revestido con el mayor rigor posible $y$, ello solo es alcanzable en la medida en que a la par con la construcción de los objetivos y planteamientos que representan la primera parte de ellos, de manera paralela, debe comenzar a desarrollarse el estudio sobre las hipótesis y la metodología, que también revisten igual escala de importancia en el evento, que hacen trascender la primera etapa, pero igualmente, enriquecen la capacidad de formulación que implica la vinculación de la empresa en el laboratorio. Enfatiza Ochoa ${ }^{1}$ (2008, p. 22):

Yo veo el potencial de la alianza universidad y empresa en que aprendamos

\footnotetext{
El académico y expresidente del Grupo Empresarial Antioqueño narra la experiencia vivida tras varias décadas dedicadas a la doble tarea de universitario y dirigente empresarial, ambas de tiempo completo.
}

a trabajar en equipo. Lo importante no son las asesorías, ni que la universidad le venda servicios a la empresa, ni que la empresa le haga donaciones a la universidad. Todo eso es superficial a la hora de la verdad. Lo verdaderamente importante es que se puedan hacer equipos conjuntos, trabajándole a proyectos de desarrollo tecnológico sofisticado.

El laboratorio constituye el encuentro de ambas divisiones en la medida en que corroboran o reflejan las pretensiones que en principio comprendieron la etapa de elaboración y formulación, que terminan dirigidas de manera simultánea para que puedan presentarse otros argumentos que en el escenario de las lógicas esperadas del laboratorio no necesariamente espera que se comporten de forma definida. Es por ello que, para poder acusar la tendencia de formalizar el paso de solo disposición de proyectos al laboratorio, debe contemplarse dicho ámbito de trabajo. Analiza Pérez (2008, p. 111):

Si miramos lo que ocurre ahí fuera podemos ver que esta primera consecuencia de la complejidad se está abriendo paso. Así, en el mundo académico es cada vez más frecuente la creación de equipos transdisciplinares para llevar a cabo una investigación. Una práctica que las grandes organizaciones internacionales ya están implementando para estudiar los problemas que tienen que afrontar. Pero que es poco frecuente en el mundo profesional, en el que todavía pesa mucho la mirada monodisciplinar, y en el que se sigue trabajando con base en el "informe del economista" o "la propuesta del arquitecto", en vez de integrar las distintas visiones en un esquema conjunto. 
La incubación en el laboratorio debe tener un proceso de filtro para poder auspiciar escenarios de salida desde donde se advierte que los análisis y revisiones hechas por medio de diversas pruebas, que implican para los comprometidos en la investigación la continuidad o revisión de los hechos contemplados inicialmente, de la propia etapa de análisis en el laboratorio y de la posibilidad de redirigir las premisas iniciales de la investigación hacia otros frentes con el propósito de obtener elementos de mayor impacto y relevantes. Postula Dávila (2008, p. 68):

Una fuente importante que nos permite entender la realidad empresarial es la preparación de casos. Dentro del mundo académico se escuchan voces que la consideran una pérdida de tiempo. Desde el punto de vista de rigor están en lo cierto, ya que muchas veces tienen serias deficiencias por este lado. Pero el objetivo de los casos no es la pureza científica, sino aprender e identificar nuevas tendencias, que después se pueden explorar con el rigor de un buen diseño de investigación. Escribir buenos casos no es fácil y requiere tiempo y una buena dosis de conocimiento y dedicación.

El laboratorio de investigación empresarial termina cumpliendo el papel de mecanismo de evaluación de todo el proceso llevado a cabo en la investigación, hecha para la empresa. Además, establecería claramente los hechos trascendentales no tenidos en cuenta o, de ser el caso, sugerir revisión a las etapas o pautas iniciales, más que para reconsiderar el proyecto, con el fin de hacer escrutinio en detalle a los parámetros que pudieron haberse contemplado y que no fueron tenidos en cuenta. Así, se cumplen los criterios de laboratorio. Establece Abello (2007, p. 210):

La nueva dinámica de producción del conocimiento es un factor definitivo a la hora de establecer las estrategias para sustentar la transferencia y apropiación de este como resultado de la investigación y desarrollo (I+D) que se realiza en las universidades con vocación investigativa. En la actualidad uno de los sellos distintivos en la producción del conocimiento tiene que ver con el contexto de su "aplicación"; en este sentido, la generación de conocimiento está directamente relacionada con la intención de ser útil para alguien, ya sea la industria, el Gobierno o la sociedad.

El extender mecanismos y procesos de aplicación a los resultados obtenidos en cada paso y de manera completa en los proyectos de investigación, debe ser una etapa consultada con la estructura de dirección y gestión de conocimiento existente entre las partes involucradas, empresa y universidades. Ello reviste la oportunidad de ubicar el contenido del trabajo en un espacio de permanente estudio y revisión a disposición entre ellas, en cuanto a la perspectiva de acoger las iniciativas de investigación en un encuentro de trabajo con fines de aplicación. Establecen Marzo et al. (2008, p. 49):

Entre los clientes de las universidades destacan las empresas, ya que las relaciones que potencialmente se pueden establecer entre ambos agentes son potencialmente generadoras de beneficios para ambas partes. La obtención de dichas ventajas permitiría a estos agentes enfrentarse con más fortaleza y 
seguridad a los retos del futuro. Así, las universidades podrían incrementar la obtención de recursos económicos, realizar intercambios de personal y de conocimientos, obtener información sobre las demandas empresariales en cuanto a investigación y capital humano, mejorar la producción científica, etc. Del mismo modo, las empresas obtendrían también ventajas como el flujo de personal cualificado, la disposición de un soporte técnico y cualificado, e incluso, mejorar su imagen ante sus clientes $\mathrm{u}$ otros stakeholders.

Es precisamente la observación uno de los criterios que más se olvida a la hora de promover factores que podrían mejorar el desarrollo del proyecto dirigido hacia el laboratorio. El seguimiento a la conducta y desempeño de las actividades que son perseguidas hace parte del diseño que debe desarrollarse o prototipo que ha de ilustrarse a la hora de evaluar los resultados obtenidos en el proyecto. En este sentido, el trabajo a consultar en el laboratorio requiere girar en estos frentes para que efectivamente se pase de la reiterada evaluación a la básica demostración. Explican Schmal et al. (2010, p. 120):

Esta desvinculación, si bien puede ser válida bajo una manera de ver la ciencia y la tecnología, la tendencia apunta hacia un mayor relacionamiento universidad-empresa de modo que las ideas/proyectos que emerjan de los investigadores estén destinados a resolver problemas reales no resueltos a la fecha.
En el laboratorio deben aplicarse simultáneamente herramientas, elementos y prototipos que igualmente arrojan perspectivas no auspiciadas inicialmente en el proyecto. En dicho sentido es que se revitalizan, además de las premisas orientadoras del proceso, el fundamento básico, para lo cual se esgrime que debe contarse con un laboratorio en el que los propósitos sobre los que es estructurado puedan tener una matriz de resultados, de evidencias, demostraciones y diseños, que puedan recrear el perfil de dicha instancia. Argumenta Ortega (2009, p. 9):

Encontrada la justificación para cambiar los medios de investigación en administración por ideas y herramientas más acordes con las tendencias generales de la ciencia, surge la necesidad de constituir una entidad interdisciplinaria que analice los problemas desde perspectivas que intenten separar los problemas de su entorno y que los resuelva con métodos que han aprobado su efectividad en campos muy distintos de la actividad humana.

El poder llegar a dicho nivel por parte de los involucrados en la investigación constituiría el principio para que los ejercicios establecidos al margen de estas instancias puedan ser evaluados desde otros frentes como la modelación, las pruebas o el trabajo de campo, para, entre otras, conocer los grados de respuesta o la capacidad que tienen de responder a parámetros iniciales destacados o en otros escenarios 
robustecidos para poder obtener elementos de fondo que puedan servir a las empresas con las que efectivamente se pactó el impacto del proyecto.

\section{Aplicación y formulación de los hallazgos desde los laboratorios de investigación empresarial}

El laboratorio se define conforme a los criterios que se establecen por las partes en cuanto a las intenciones de evaluación y revisión que se tienen previstas desarrollar en este. La secuencia de análisis, pruebas y ajuste depende de la expectativa que se tiene con el objeto de estudio y trabajo de campo a considerar. No obstante, es necesario que a medida que se trabaja con relación al tema de estudio, igualmente sea atendido el análisis concerniente a la hipótesis y metodología, debido a la trascendencia de dichos componentes en el laboratorio. Agrega Ferrás (2010, p. 143):

La estrategia de innovación de un país, al igual que la estrategia competitiva de una empresa (como hemos visto ya), no tienen por qué consistir en un pesado tomo de varios capítulos de análisis, otros tantos de prospectiva y diagnosis, y finalmente un despliegue de varios centenares de propuestas. No tiene por qué ser una propuesta de desarrollo de la industria aeronáutica o biotecnológica. La estrategia, la forma básica de competir de las naciones, puede ser algo tan simple como detectar líderes y dotarlos de recursos. Esa es, sin duda, una gran estrategia.
La naturaleza del laboratorio tiende a complicarse por lo menos en lo que respecta a la capacidad de revisión a la que deben someterse la investigación o el proceso, conduciendo este espacio a otros escenarios como el de las consultorías o estudios, lo que si bien no es contraproducente, debe tenerse en cuenta en el proceso. La base para poder llevar los casos e investigaciones a concurso de laboratorio debe explícitamente estar sujeta a premisas de análisis o fundamentos que revistan la composición de hipótesis y metodologías. Indica Mondragón (2009, p. 22):

No obstante, en forma paralela está ocurriendo la situación de que la transferencia de los productos de la investigación hacia el sector productivo sigue siendo un problema por resolver. En el campo de empresa, sobre todo en el de las grandes multinacionales de Estados Unidos, Europa y Japón, hay un aspecto interesante porque ellas tienen sus propios centros de investigación y desarrollo. Y allí se investiga desde la parte más básica en química, física y biología, y se logra la cadena con la investigación correspondiente en ingeniería hasta llegar al producto final. Hay empresas que, presionadas por la avalancha que se les viene encima por los tratados de libre comercio, están tomando medidas en el mejor sentido y están empezando a invertir en investigación. Saben que si no cambian, desaparecen.

Es el compromiso de las empresas en cuanto a la profundidad con que se quiera llevar a cabo el ejercicio del laboratorio, la razón que impulsa a analizar las condiciones de ellas desde una mirada de aporte y contribución, y no de manera 
simple, un traslado de información de una fuente a otra. De ahí que es importante para las partes establecer las bases sobre las cuales apoyarían el proceso de pruebas y revisión en estos contextos, a la hora de orientar un trabajo de análisis más estilizado y consecuente con las expectativas básicas definidas en investigación e innovación. Expresa Pulido: "Una receta simplista para la innovación consiste en repartir responsabilidades. Estas últimas deben invertir suficientes recursos en laboratorios y centros de I+D+i" (2005, p. 17). Y complementa Sanabria (2005, p. 79):

Sin embargo, la empresa es tan solo "un tipo" particular de organización. Por lo cual, este terreno, así como el de la gestión organizacional, se encuentra aún frente a múltiples y variadas formas de desarrollo conceptual, teórico y práctico, sobre la base de organizaciones tan diversas como las de salud, educativas, estatales y civiles, entre otras.

El resultado en los procesos de análisis, experimentación, evaluación, pruebas y simulación llevados a cabo en el laboratorio de investigación empresarial está sujeto a las perspectivas que de manera colegiada establecen las partes. Ya no solo depende del grueso de aspectos que han revestido el proceso, ahora el tema se traslada a la revisión del contenido en materia de calidad y de impacto, del cual se espera que las empresas y los grupos de investigación saquen el máximo provecho. Comentan Anlló y Ramos (2008, p. 8):

De igual forma, esta decisión por parte del sector privado se ve acompañada por una internacionalización reflejada en la conformación de redes de investigación globales (integradas por laboratorios públicos, universidades y actores privados alrededor del globo), así como un notable aumento en la movilidad internacional de los científicos (dando lugar a fenómenos como el brain drain ${ }^{2}$.

La protección a la información, la determinación de la que puede publicarse y la disposición de difundir solo una parte de esta, representa el esquema de negociación a la que en gran medida deben ser extendidos los análisis estimados en el laboratorio. Sin embargo, la idea es poder contar con la continuidad del trabajo en la medida en que se puedan desarrollar conforme a lo obtenido en el laboratorio otras fuentes de análisis, que revitalicen la convergencia de intereses en el tiempo y que se traduce para las partes en la posibilidad de orientar trabajo estratégico. Complementan García y Sureda (2001, p. 309):

Todo ello se fundamenta al comprender que la ciencia constituye hoy objeto de investigación de primer orden, debido, esencialmente, al papel tan importante que le corresponde en la sociedad contemporánea. Convertida en columna vertebral del organismo social, es decir, de su economía, de su política y su cultura, de sus funciones y fuerzas motrices, se convierte en un elemento indispensable en la sociedad del nuevo milenio.

El impulsar el trabajo desarrollado en el laboratorio hacia otros frentes está sujeto a la capacidad de gestión de

2 Fuga de cerebros 
conocimiento inserta en las partes convocantes en los proyectos de investigación empresarial. La lucidez que se tenga al respecto responde a las metas que las partes hayan definido comunes y diferenciales de manera simultánea. Dicha traza es necesaria que sea definida, por cuanto la labor en la que están inmersas las empresas y los grupos de investigación así lo indica. Sugieren Montejo y Bravo (2010, p. 23):

Es también muy conveniente que los participantes en un proyecto innovador posean un buen conocimiento de la organización de la empresa y de los individuos o unidades, que pueden aportar puntos de vista valiosos para el proyecto, así como de los que tienen poder para tomar decisiones en caso de dificultades o posibles disyuntivas en el desarrollo del mismo. La propia estructura organizativa debe ser abierta para facilitar la sinergia con los proyectos innovadores; en ella es fundamental el papel de los supervisores, el primer nivel de "dirección”, responsable no solo de su propio trabajo, sino también del resultado del de los supervisados, por esta razón la habilidad tecnológica es todavía muy importante en este nivel de dirección.

Es el punto de quiebre en el cual se divide la proyección de las partes con los respectivos intereses expuestos, lo que refrenda el papel del laboratorio. En todo el proceso como tal se tienen límites, que no necesariamente son conversados o estimados de manera profunda. Sin embargo, cuando las metas comienzan a concretarse y estas a plantear posibilidades de resultados importantes para las partes es cuando se espera que el vínculo se estreche más, aunque por la lógica de interacción, al agotarse los propósitos iniciales estos deban evaluarse hacia delante (ver cuadro 2).

Cuadro 2. Ejemplos de eventos de laboratorio

\begin{tabular}{|c|l|}
\hline \multicolumn{1}{|c|}{ Autor } & \multicolumn{1}{c|}{ Evento } \\
\hline Gainza, E. (2003, p. 7) & $\begin{array}{l}\text { La Universidad de Stanford, cuna de 25 Premios Nobel y referente indiscutible en ciencia } \\
\text { y tecnología, fue la creadora en } 1951 \text { del primer parque tecnológico del mundo, el Stan- } \\
\text { ford Research Park. Este se convirtió en el corazón de Silicon Valley, el entorno más para- } \\
\text { digmático de las nuevas tecnologías en el ámbito mundial. }\end{array}$ \\
\hline Bernal, C. (2011, p. 16) & $\begin{array}{l}\text { Tony Blair, ex primer ministro inglés, propuso la enseñanza de crear y desarrollar empre- } \\
\text { sas en los colegios de empresarios: "La razón es simple: se ha apagado en su país la llama } \\
\text { de la iniciación empresarial” (El Tiempo, Editorial, 1998). }\end{array}$ \\
\hline
\end{tabular}

Es importante que de nuevo se retome el rumbo de la consecuencia lógica que traen los procesos de inmersión en investigación, que comúnmente son destacados en los trabajos realizados en el laboratorio pero que tienen como premisa orientar hacia la consolidación de los procesos ya existentes o la disposición a encontrar caminos para apoyar diversos intereses e iniciativas presentadas por las empresas, que de la mano con los grupos de investigación pueden tener respaldo en la medida en que se atiendan metas comunes de manera general. Argumentan Castro y Vega (2009, pp. 77 y 78):

Es verdaderamente importante equipar a las universidades y a las empresas con herramientas que les permitan familiarizarse mutuamente por medio de un mejor conocimiento de lo que necesitan y de lo que son capaces de hacer en conjunto. Por lo tanto, se requiere mucho más 
información respecto a la oferta científica y tecnológica de la Universidad, así como acerca de las necesidades reales de conocimiento en el sector empresarial. El mejoramiento de los flujos de información ayuda, con el tiempo, a incrementar la legitimidad de los mecanismos de cooperación y reconocerlos como una estrategia de desarrollo importante, a través de la cual los actores participantes obtienen ganancias sustanciales.

El concepto de laboratorio de investigación empresarial pretende precisamente trabajar de cara a las oportunidades o situaciones que deben enfrentar las empresas y que no alcanza a los cometidos proveídos por las consultorías o los estudios específicos. Por ello, el compulsarlo bajo dicha denominación requiere además de elementos y fundamentos, prestancia para estructurar, elaborar y ejecutar distintas pruebas, conforme al análisis en particular planteado, para que la empresa pueda tener idea o razones con la finalidad de considerar lo obtenido en dichas etapas como objetos de suma importancia para su actividad.

\section{Conclusiones}

El laboratorio de investigación empresarial postulado como premisa de trabajo interinstitucional recoge varias de las experiencias exitosas que se han considerado en el mundo, respecto al papel que deben cumplir las universidades y los grupos de investigación cuando se trata de analizar temas de orden transversal como los análisis de corte empresarial, desde los cuales siempre se espera que las empresas, además de recibir un documento, tengan elementos de fondo con los cuales poder actuar y tomar decisiones conforme a sus situaciones.

La expectativa creada con el laboratorio estriba en la posibilidad de llevar a revisión la estructura sobre la cual se guían las empresas en diferentes contextos, con la finalidad de establecer bases y elementos en las que ellas trasiegan y a partir de ellos poderse orientar de conformidad con las circunstancias que aparezcan, y entregar u ofrecer alternativas de consideración. No obstante, esto depende de la sintonía que se tenga con el proceso de laboratorio, que dada su magnitud, requiere un fundamento permanente y no propiamente consideraciones esporádicas de hecho.

El iniciar dichas etapas de manera simultánea permite orientar no solo la proyección establecida de la investigación, sino determinar la complementariedad o dispersión que la metodología acogida en principio podría establecer. El punto de enlace de dicho proceso se da cuando a la par del inicio de los proyectos de investigación o trabajos de interés para las empresas, y de acoger los fundamentos establecidos en los objetivos, se tomen de manera paralela las referencias o postulados que de orden hipotético o metodológico se asumen como fuente de trabajo.

El resultado de los trabajos no termina en manos de las empresas objeto de interés, y tampoco son claras las razones del por qué no se continúa trabajando con ellas a partir precisamente del grueso de hallazgos, que son los que claramente deben ir a proceso de estudio y revisión, para, entre otras razones, efectivamente considerar que lo abonado en el proceso tiene un sentido superior al del cumplimiento de unos objetivos provistos, como lo es el proveer fundamentos a 
las empresas que con su disposición ofrecieron tiempo para desarrollar el trabajo.

\section{Referencias}

I. Abello, L. R. (2007). Factores claves en las alianzas universidad-industria como soporte de la productividad en la industria local: hacia un modelo de desarrollo económico y social sostenible. Investigación y desarrollo, 15(1), 208-225.

2. Abello, R.; Dacunha, C. y Páez, J. (1991). Reflexiones sobre cooperación universidad-empresa-Estado. Ingeniería \& Desarrollo, 151-166.

3. Anlló, G. y Ramos, A. (2008). Innovación, estrategias empresariales y oportunidades productivas de las firmas extranjeras en Brasil y Argentina. Comisión Económica para América Latina y el Caribe (Cepal), 1-42.

4. Bernal, H. C. (25 de octubre de 2011). Laboratorio de empresa en la educación empresarial. Escuela de Administración de Empresas (UPTC - Tunja). Instituto para la Investigación Educativa y el Desarrollo Pedagógico, IDEP - Alcaldía Mayor de Bogotá. Obtenido de henrycbc@yahoo.com; http://desnet. uptc.edu.co/labstar

5. Canada, A. (2010). Mapas de oportunidades: representar gráficamente el futuro en tiempos de incertidumbre. Harvard Deusto Business Review, 41-48.

6. Casanovas, I. (2005). La didáctica en el diseño de simuladores digitales para la formación universitaria en la toma de decisiones: un modelo teórico metodológico de diseño de simuladores de toma de decisiones basado en indicadores didácticos. LIE-FI-UBA. Revista de Informática Educativa y Medios Audiovisuales, 2(6), 17-34. Obtenido de liema@fi.uba.ar
7. Castro, M. E. y Vega, J. J. (2009). Las relaciones universidad-entorno socioeconómico en el espacio iberoamericano del conocimiento. CTS, 4(12), 71-81.

8. Dávila, A. (2008). ¿Cómo se investiga? IESE Business School, 61-69.

9. Fracica, N. G. (2009). La financiación empresarial y la industria del capital de riesgo: estructura y conceptos. Revista EAN, 66, 31-66.

Io. Ferrás, X. (2010). Innovación 6.0: el fin de la estrategia. Barcelona, España: Plataforma Editorial.

I I. Galán, J. I. y Sánchez, M. J. (2004). Coherencia entre el cambio estratégico y organizativo: nuevas formas de organización. Cuadernos de economía y dirección de la empresa, 19: 141-176.

I 2. García, C. J. y Sureda, T. (2001). La ciencia: un enfoque dialéctico-materialista de su devenir. Instituto de Ciencias Básicas y Preclínicas Victoria de Girón. Rev. Cubana Invest Biomed, 20(4), 302-310.

I3. Gainza, E. (2003). Universidad-empresa, un equipo con futuro. Revista de la Red de Parques Tecnológicos de Euskadi, 21, 1-32.

I4. Instituto Internacional de la Unesco para la Educación Superior en América Latina y el Caribe, Iesalc (2008). Transformaciones sociales y desafíos universitarios en América Latina. Educación Superior y Sociedad, 13(1), 1-144. Obtenido de iesalc@unesco.org.ve; www. iesalc.unesco.org.ve

I 5. Marzo, M.; Pedraja, M. y Rivera, P. (2008). Un modelo de relaciones empresa-universidad. Revista Europea de Dirección y Economía de la Empresa, 17(1), 39-56.

I6. Miyahira, J. (2009). La investigación formativa y la formación para la 
investigación en el pregrado. Rev. Med Hered, 20(3), 119-122.

I7. Mondragón, P. F. (2009). El problema está en cómo valorar la propiedad intelectual. Universidad, Empresa y Estado, 2(3), 1-43.

I8. Moreno, J. C. y Ruiz, N. P. (2009). La educación superior y el desarrollo económico en América Latina. Estudios y Perspectivas, 106, 1-45.

I9. Montejo, M. J. y Bravo, J. A. (2010). Cotec. La innovación en sentido amplio: un modelo empresarial. Análisis conceptual y empírico. Innovación práctica. Fundación Cotec para la Innovación Tecnológica, 1-69

20. Ochoa, J. C. (2009). Hacia una cultura del emprendimiento, la innovación y la asociatividad para la competitividad y el desarrollo de la región y del país. Universidad, Empresa y Estado, 2(3): 22.

2r. Ondagueti, J. C. (2001). Parques científicos y tecnológicos: los nuevos espacios productivos del futuro. Investigaciones Geográficas, 25, 95-118.

22. Ortega, F. F. et al. (2009). Protocolo de implementación del LMyS. Implementación del laboratorio de modelamiento y simulación. Estudios Empresariales para la Perdurabilidad, 1-36.

23. Pérez, R. A. (2008). Estrategar en un mundo complejo. Cinco consecuencias en la práctica profesional. Fisec: Estrategias, 4(10), 85-131.

24. Pérez, J. A. (2009). Universidad y gerencia en el medio internacional. Investigación, innovación y competencias, 17, 156-182.

25. Pulido, A. (2005). La innovación en el siglo XXI. Centro de Predicción Económica (Ceprede)-IBM. Universidad Autónoma de Madrid, Facultad de Ciencias Económicas y Empresariales, 1-27.
26. Renzulli, J. S. (2010). El rol del profesor en el desarrollo del talento. Reifop, 13(1). Consultado el 25 de septiembre de 2012. Obtenido de http://www.aufop.com

27. Rodríguez, G. D. y Betancur, J. H. (2009). Ejercicio de transversalidad y responsabilidad social. Una experiencia de formación integral. Institución Universitaria de Envigado Conciencia Empresarial. Facultad de Ciencias Empresariales, 3, 37-63.

28. Sanabria, M. (2005). El pensamiento organizacional estratégico: una perspectiva diacrónica. Innovar, 24, 59-81.

29. Schmal, R.; López, S.; Cabrales, F. y Acuña, D. (2010). Modelado de procesos de negocio para la gestión de patentes en universidades. Información Tecnológica, 21(6).

30. Segarra, E. (2010). ¿De dónde proceden las ideas? Las fuentes de la innovación en la empresa. Harvard Deusto Business Review, 51-56.

3I. Svarzman, G. (2007). Empresas transnacionales: sus estrategias de investigación y desarrollo y el papel de Argentina y el Mercosur. Comisión Económica para América Latina y el Caribe (Cepal), 1-45.

32. Yusuf, S. (2007). University-industry links, policy dimensions. En Shahid Y. y K. Nabeshima (Eds.), How Universities Promote Economic Growth, Directions in Human Development. Washington, D. C.: Banco Mundial.

33. Yusuf, S. y Nabeshima, K. (Eds.) (2007). How Universities Promote Economic Growth. Washington, D. C.: Banco Mundial. 